IRA-International Journal of Applied Sciences ISSN 2455-4499; Vol.06, Issue 02 (2017)

Institute of Research Advances

Pg. no. 55-61

https://research-advances.org/index.php/IRAJAS

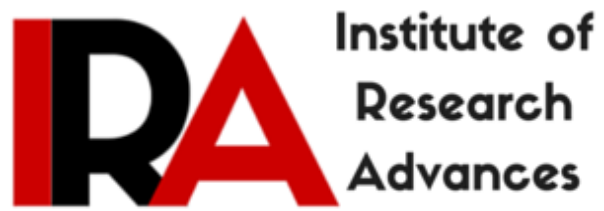

\title{
Investigations of Current Generated in Construction of Polarization in Vectra A, Liquid Crystal Copolyester
}

\author{
Sapna Kalia ${ }^{1}$ and Rajesh Kalia ${ }^{2}$ \\ ${ }^{1}$ Department of Physics, Maharishi Markandeshwar University, \\ Mullana (Ambala)-133 207, Haryana, India. \\ ${ }^{2}$ Department of Applied Science, UIET, Kurukshetra University, \\ Kurukshetra-136119, Haryana, India.
}

Type of Review: Peer Reviewed.

DOI: http://dx.doi.org/10.21013/jas.v6.n2.p4

\section{How to cite this paper:}

Kalia, S., \& Kalia, R. (2017). Investigations of Current Generated in Construction of Polarization in Vectra A, Liquid Crystal Copolyester. IRA-International Journal of Applied Sciences (ISSN 2455-4499), 6(2), 55-61. doi:http://dx.doi.org/10.21013/jas.v6.n2.p4

(C) Institute of Research Advances

\section{(cc) EY-NC}

This work is licensed under a Creative Commons Attribution-Non Commercial 4.0 International License subject to proper citation to the publication source of the work.

Disclaimer: The scholarly papers as reviewed and published by the Institute of Research Advances (IRA) are the views and opinions of their respective authors and are not the views or opinions of the IRA. The IRA disclaims of any harm or loss caused due to the published content to any party. 


\section{ABSTRACT}

The Thermally Stimulated Polarization Current (TSPC) is used to study the dielectric behavior of Vectra A, liquid crystal copolyester. The TSPC spectra of samples of Vectra A for various polarizing fields (3.8 $\mathrm{kV} / \mathrm{cm}-19.2 \mathrm{kV} / \mathrm{cm}$ ) have been investigated. A dipolar relaxation is observed in the low temperature region $\sim 30{ }^{\circ} \mathrm{C}$ due to orientation of naphthalene groups present in Vectra $A$ which is termed as peak- $P_{1}$. In the higher temperature region above $110^{\circ} \mathrm{C}$, the dipolar and space charge peaks are in composite form. TSPC cycle is performed to distinguish the dipolar and space charge peaks. Also, the TSPC spectra of annealed and doped samples of Vectra A are studied. The results obtained from TSPC spectra are compared with TSDC spectra of samples of Vectra A.

Key words: Thermally Stimulated Polarization Current (TSPC), Vectra A, poling filed, annealing, doping

Introduction: In Thermally Stimulated Polarization Current (TSPC) technique, the current is generated in construction of polarization. This technique is a complimentary to Thermally Stimulated Discharge Current (TSDC) technique where current is generated when destruction of polarization takes place [1]. The relevance of the TSPC technique becomes important because the comparison between processes of generating current during construction of polarization and during destruction internal polarization will provide useful results which otherwise, cannot be obtained from either of them [2]. Although TSP current suffers from an unavoidable superposition by the internal conductance, it has superior aspect as it avoids overheating of sample and eliminates the search for poling temperature. The TSPC technique gives an idea of the temperature at which conduction current becomes significant. This is necessary because after the start of conduction phenomenon, the stored charges in the dielectric are quickly neutralised thus preventing the subsequent TSPC and TSDC from its proper functioning [3-4].

The aim of the present work is to study the dielectric relaxations in Vectra A by investigating the TSPC spectra at different polarizing fields. The effect of annealing and doping is also discussed.

Material and Method: The Vectra A, ivory in color, is procured in the form of rod having diameter 18 millimeter from Good fellow, England. The glass transition temperature of Vectra A is around $110{ }^{\circ} \mathrm{C}$ and it melts at $280{ }^{\circ} \mathrm{C}$. It is copolyester of polyhydroxybenzoic acid, PHBA and poly naphthoic acid, PHNA. In Vectra A, the incorporation of 2, 6-naphthalylene moieties in 4-hydroxybenzoic acid results in the structural defect and reduction in rigidity of chain. The pristine, annealed and doped samples of Vectra A having thickness $1.3 \mathrm{~mm}$ were metalized on both sides by silver paste and held in a sample holder. The sample holder was suspended inside a properly earthed aluminum chamber of the furnace. After reducing the transient current to almost negligible value at room temperature, the sample was heated at linear rate $\left(2{ }^{\circ} \mathrm{C} / \mathrm{min}\right)$ with simultaneous application of constant dc electric field $\left(\mathrm{E}_{\mathrm{P}}\right)$ in series with a sensitive electrometer (Kiethley 6514) to measure the TSP currents. The slow heating rate is required to prevent temperature gradient within the sample, temperature difference between sample and surroundings as well as for obtaining a fine resolution in TSPC spectra [2]. If the sample is short circuited for sufficient time to allow the spurious polarization to decay, it may altogether eliminate the dipoles in random motion. In the present work this process has been followed while carrying out the repeated TSPC cycles. Between two successively repeated cycles, the sample was allowed to cool from maximum temperature to room temperature and was kept for about 30 minutes to allow the entire relaxation mode to become almost saturated at that point.

\section{Results and Discussion}

The TSPC spectra of the Vectra A samples for various polarizing fields $(3.8 \mathrm{kV} / \mathrm{cm}-19.2 \mathrm{kV} / \mathrm{cm})$ have been shown in Figure 1. Two current peaks are observed in TSPC spectra of Vectra A samples viz. current peak $\left(\mathrm{P}_{1}\right)$ which occur $\sim 30{ }^{\circ} \mathrm{C}$ and current peak $\left(\mathrm{P}_{2}\right)$ appearing $\sim 110{ }^{\circ} \mathrm{C}$. The magnitude of both 
$\left(\mathrm{P}_{1} \& \mathrm{P}_{2}\right)$ peaks increases with the increase in polarizing field. The current peak $\left(\mathrm{P}_{2}\right)$ shifts towards higher temperature with the increase in polarizing field. A steady increase in TSP current is observed in high temperature region $\left(\sim\right.$ above $\left.175^{\circ} \mathrm{C}\right)$. These results are in accordance with the non-isothermal charging mechanism of a polymer in which the polarizing current is assumed to have two components behaving differently as a function of temperature. Dipolar orientation as a transient process which results in the formation of a peak and the conduction current due to the motion of equilibrium charge carriers increasing continuously with temperature.

The charging current arises from the orientation of naphthalene group appears in the form of TSPC peak $\left(\mathrm{P}_{1}\right)$. Similar peaks are observed in Vectra $\mathrm{B} \sim 15{ }^{\circ} \mathrm{C}$ and in Vectra $\mathrm{E} \sim 35{ }^{\circ} \mathrm{C}$ by various workers [4-6]. This peak occurrence in Vectra series is attributed to a common linkage of ester group [5]. The peak $\left(\mathrm{P}_{2}\right)$ is attributed to orientation of main chain segment. The increase in magnitude of the TSPC peaks is due to occurrence of uniform bulk polarization [7].

The shift in dipolar peak $\left(\mathrm{P}_{2}\right)$ towards higher temperature with the increase in polarizing field is due to the presence of spacers connecting side chain groups to main chain group. These spacers are flexible in length [8]. Thus, with the increase in polarizing field at higher temperature, length of spacers is reduced resulting in orientation of side chain groups at elevated temperature and shifting of peak occur [9].

In TSPC, peak $\left(\mathrm{P}_{2}\right)$ is fused with space charge relaxation and it is not as well defined as in case of TSDC spectra ( $\alpha$-peak). These composite peaks can be separated by repeating the TSPC cycles. Dipolar peaks get eliminated in second cycle as saturation polarization occurs and none of dipoles are left for orientation but space charge peak remains as illustrated in Figure 2.

Vanderschurren and Gasiot [3] reported that the TSP currents will be characterized by peaks roughly similar to those appearing in TSDC measurements but they will obviously appear in a reverse direction, at least in the beginning of the spectrum. Simmons et al. [9] and Devaux et al. [10] proposed a trapping model according to which, the TSPC peaks without reversal and similar to the TSDC peaks can also be observed, provided at least one of the electrodes is non-ohmic. The magnitude of the TSPC peak is large as compared to the TSDC peak at similar condition of poling field $\left(\mathrm{E}_{\mathrm{P}}\right)$ which is attributed to superposition of true conduction currents and higher noise level in TSPC spectra and buildup during the polarizing period of ionic space charge which results in reducing the forming field [3]. 


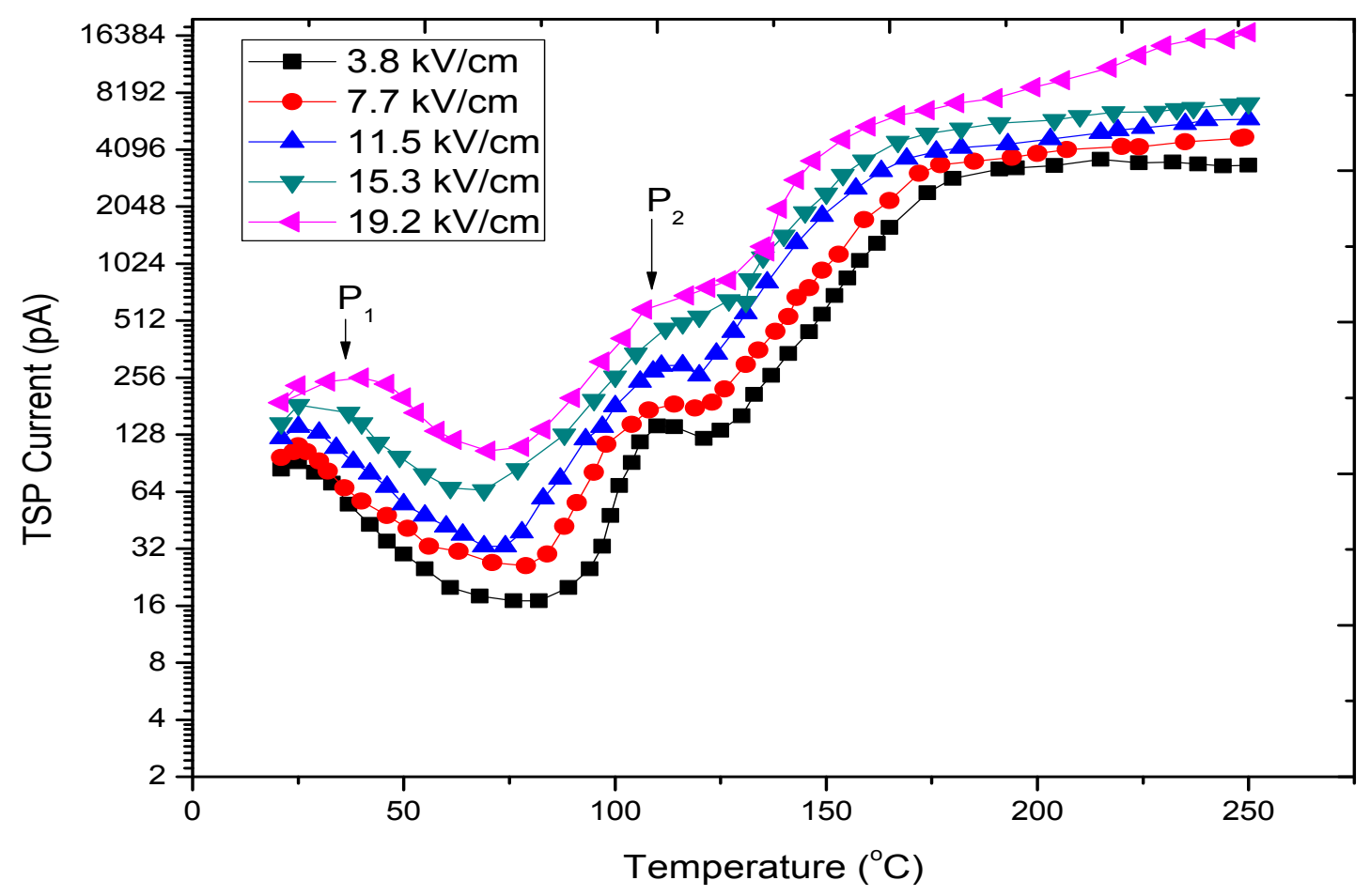

Figure 1: The TSPC spectra of Vectra-A samples for different polarizing fields $(3.8 \mathrm{kV} / \mathrm{cm}-19.2$ $\mathrm{kV} / \mathrm{cm})$.

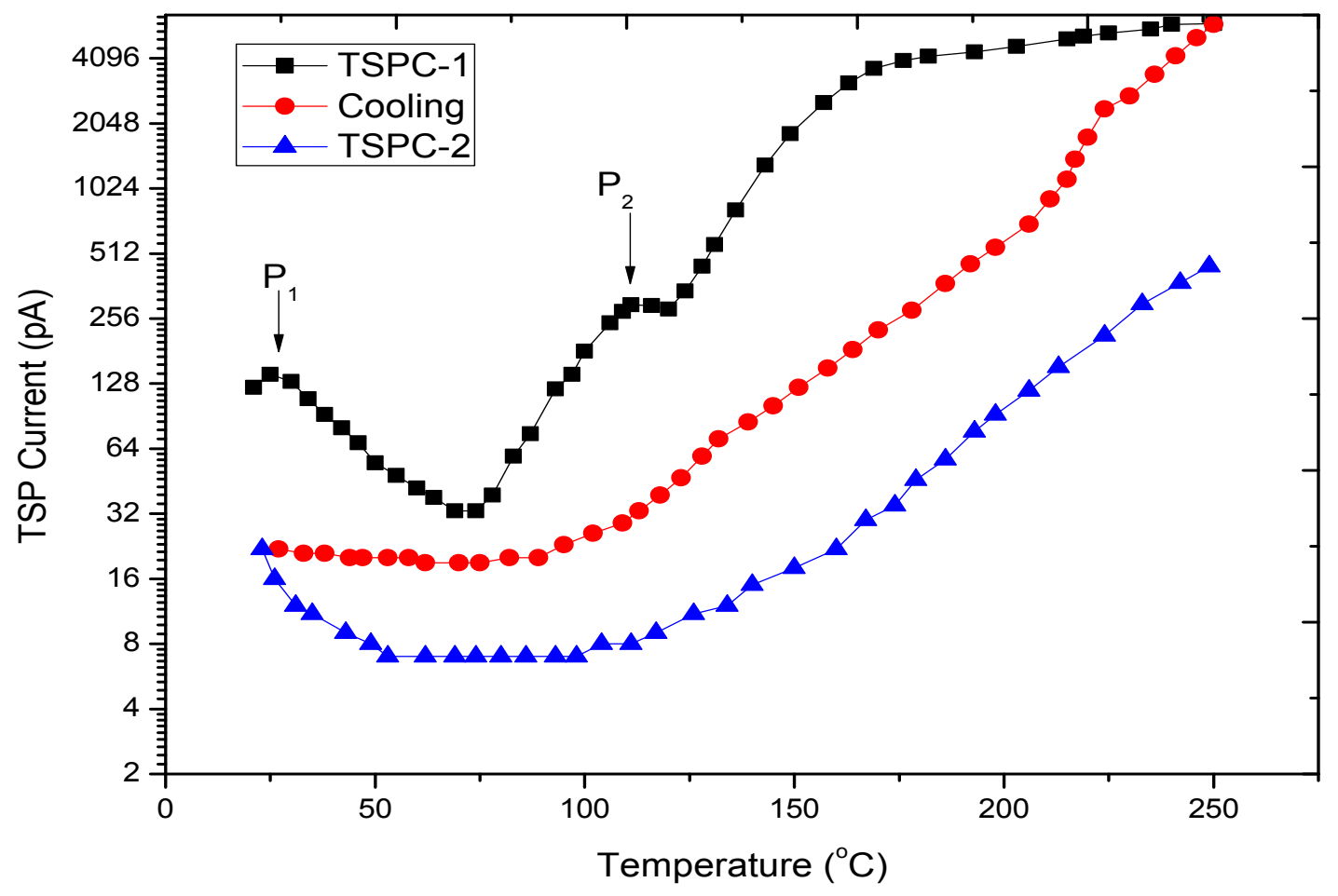

Figure 2: Repeating heating-cooling-reheating spectra of Vectra-A sample for poling field $\left(E_{P}\right)=11.5$ $\mathrm{kV} / \mathrm{cm}$. 


\section{Effect of Annealing}

Annealing is a pre-treatment given to polymeric samples to obtain its improved thermal stability and crystallinity [1]. The annealing of the polymers enhances their sensitivity of cooperative relaxation processes, this leads to better separation of dipolar peaks [11]. Hence annealing is of great importance for polymers possessing multiple relaxation peaks [12-13]. The TSPC spectra of annealed samples of Vectra A at $100{ }^{\circ} \mathrm{C}$ for 5 hours and 50 hours and subjected to poling field $11.5 \mathrm{kV} / \mathrm{cm}$ is illustrated in Figure 3 . In low temperature region where $\beta$-peak is expected due to orientation of naphthalene group is not obtained in annealed samples as compared to pristine samples. The absence of $\beta$-peak is also observed in annealed samples of Vectra A when TSDC technique is applied [14]. The absence of $\beta$-peak is attributed to change in degree of crystallinity in annealed samples as compared to Vectra A pristine samples and hence results in reduction in dipolar relaxations in low temperature region [15-18]. As the temperature is increased there is increase in crystallinity resulting in dipolar relaxation $\sim 110{ }^{\circ} \mathrm{C}$. This relaxation is obtained due to orientation of main chain of Vectra A. Once the dipolar relaxation is over, there is dominance of conduction current as observed in Figure 3. As the annealing time is increased from 5 hours to 50 hours the dipolar peak around $110{ }^{\circ} \mathrm{C}$ shifts towards higher temperature and broadens. The increase in annealing time enhances magnitude and area of dipolar peak which is due to long range cooperative motion of the dipoles. The observations from TSPC spectra are in agreement with the observations from TSDC spectra for annealed Vectra A samples.

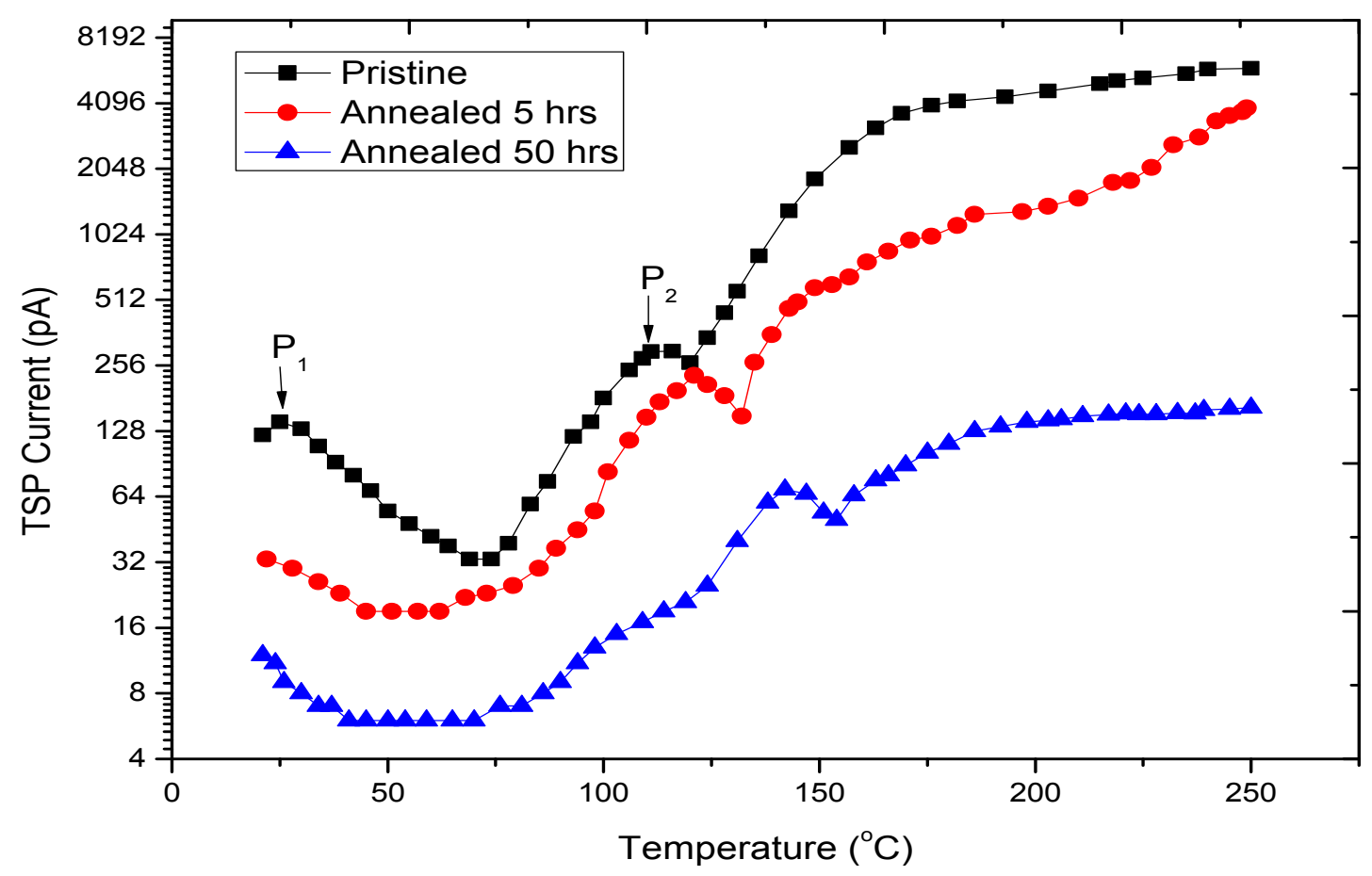

Figure 3: The TSPC spectra of Vectra-A samples under different Annealing Conditions (5 hrs and 50 hrs). 


\section{Effect of Doping}

Doping induces charge storage in polymers making them conducting. Such conducting polymers find important applications in fabrication of devices such as sensors, solar cells, rechargeable batteries and conjugated actuators [20-21]. The effect of doping of iodine on the TSPC spectra of Vectra A samples polarized at poling field $11.5 \mathrm{kV} / \mathrm{cm}$ and poling temperature $100{ }^{\circ} \mathrm{C}$ is illustrated in Figure 4. Doping of iodine in Vectra $\mathrm{A}$ is due to charge transfer (C-T) reaction. The conductivity of Vectra $\mathrm{A}$ samples doped with $4 \%$ iodine is more as compared to Vectra A samples doped with $2 \%$ iodine. Hence it is clear that conductivity of Vectra A increases with increase in doping level of iodine. It is clear from Figure 4.4 that the conductivity of Vectra A increases with the increase in temperature. The TSPC spectra of iodine doped Vectra A samples are in close agreement with the TSDC spectra.

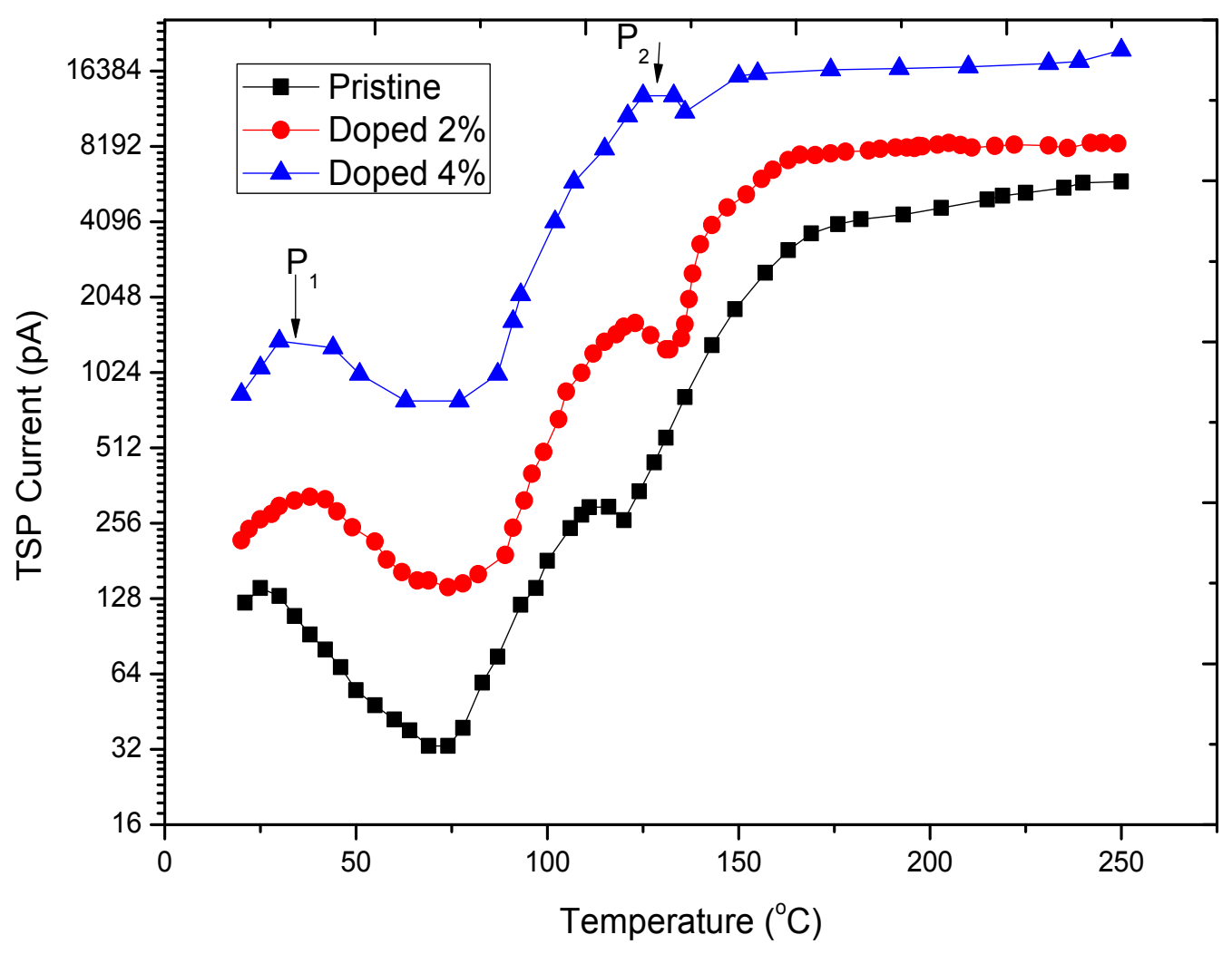

Figure 4: The TSPC spectra of Vectra-A samples under different doping concentrations (2\% and $4 \%$ by weight).

\section{CONCLUSION}

The TSPC spectra of samples of Vectra A obtained for poling fields in range $(3.8 \mathrm{kV} / \mathrm{cm}-19.2$ $\mathrm{kV} / \mathrm{cm}$ ) illustrate that in the low temperature region $\sim 30{ }^{\circ} \mathrm{C}$, there is a dipolar relaxation due to orientation of naphthalene groups present in Vectra A. These relaxations are in agreement with results obtained by TSDC. In the higher temperature region above $110{ }^{\circ} \mathrm{C}$ there is dipolar peak and space charge peak in composite form. The dipolar and space charge peaks are separated by performing the TSPC cycle second time in which only space charge peak is obtained and dipolar peak vanishes since dipoles are saturated. In the TSPC spectra of Vectra A annealed samples a shift in dipolar peak towards high temperature with increase in annealing time is observed due to long range cooperative motion. The TSPC spectra of iodine doped Vectra A samples shows enhanced dipolar and space charge relaxations which 
supports enhanced charge transfer during doping [22]. The TSPC spectra of pristine, annealed and doped samples of Vectra A are in close agreement with TSDC spectra of Vectra A samples respectively.

\section{REFERENCES}

1. A. Boresma J.V. Turnhout and M. Wubbenhorst, Macromolecules, 31, (1998) 7461.

2. D.D. Shepard and B. Twombly, Thermochimica Act., 272, (1999) 125.

3. D.S. Kalika and D.Y. Yoon, Macromolecules, 24, (1991) 3404.

4. Electrets, Ed. by G. M. Sessler, Springer-Verlag, Berlin (1980).

5. F.H.G. Bergmann and H. Finkelmann, Macromol. Rapid Commun., 18, (1997) 353.

6. J. G. Simmons and G.W. Taylor, Phys. Rev. B6, (1972) 4804.

7. J. K. Quamara and Y. Sridharbabu, Indian J of Pure \& Applied Physics. 41, (2003) 790.

8. J. K. Quamara, N. Singh and A. Singh, Macromol Chem Phys., 202, (2001) 1995.

9. J. Vanderschueren and J. Gasiot, Topics in Applied Physics, Springer-Verglas, Berlin Heidelberg, 37, (1979) 135.

10. J.K. Quamara, S.K. Mahna, S. Garg, Int. J. Polym. Anal. Charact., 15, (2010) 298.

11. K. Ueyama, Elastomers and Gels. Macromolecules, 40, (2007) 2277.

12. N.P. Gupta, K. Jain and P.C. Mahendru, Thin Solid Films, 61, (1979) 297.

13. P. Alexandrovich, F.E. Karasz and W.J. Acknight, J. Appl. Phys., 47, (1976) 4251.

14. P. Braunlich, Thermally Stimulated Relaxation in Solids, Springer-Verlag, Berlin (1979).

15. P. Braunlich, Thermally Stimulated Relaxation in Solids, Springer-Verlag, Berlin (1979).

16. P. Devaux and M. Schott, Phys. Status Solidi, 20, (1967) 301.

17. P. Huo and P. Cebe, Polymer science Part b: Polymer Physics, 30, (1992) 239.

18. R.W. Lenz, Faraday Disc. Chem. Soc., 79, (1985) 21.

19. S. Joshi and S. Radhakrishan, Thin Solid Films, 142, (1986) 213.

20. S. Kalia, J.K. Sharma, and V. Sharma, ISRN Polymer Science, (2013) 1.

21. T. D. Chang, S.H. Carr and J.O. Brittain, Polym. Eng. Sci., 22, (1982) 1205.

22. T. S. Chung, Thermotropic liquid crystal polymer., CRC Press, Ch(3), (2001). 